\title{
Application of an Adaptive Sparse-Grid Technique to a Model Singular Perturbation Problem
}

\author{
J. Noordmans ${ }^{\dagger}$ and P. W. Hemker, Amsterdam \\ Received October 18, 1999
}

\begin{abstract}
In this paper we show how, under minimal conditions, a combination extrapolation can be introduced for an adaptive sparse grid. We apply this technique for the solution of a two-dimensional model singular perturbation problem, defined on the domain exterior of a circle.
\end{abstract}

AMS Subject Classifications: 65N50, 65N55.

Key Words: Adaptive sparse grids, combination technique, singular perturbation problem.

\section{Introduction}

With multigrid-type solution methods, second order elliptic PDEs can be solved numerically with a computational effort that is proportional to the number of required nodal points. Hence, to further enhance the efficiency, the number of degrees of freedom in a discretization procedure should be reduced. For sufficiently smooth solutions the use of sparse grids [13] seems to be very efficient in this respect. One can demonstrate for solutions with bounded cross derivatives, that satisfy $\partial^{2 d} u / \partial^{2} x_{1}, \ldots, \partial^{2} x_{d}<C$ in a $d$-dimensional domain, that the accuracy of the sparse grid solution is of order $\left(h^{2} \log (h)^{d-1}\right)$ for piecewise linear interpolation, where the number of degrees of freedom is $\mathcal{O}\left(h^{-1} \log (h)^{d-1}\right)$. Such sparse grids are composed of particular sets of regular grids. The simplest way to indicate the grids involved is by saying that the sparse grid is constructed as a composite grid, which is the union of all regular grids with cell volume $\left(h_{1}, \ldots, h_{d}\right)>h$. Here, for $j=1, \ldots, d, h_{j}=2^{-n}$ for some $n \in \mathbb{N}$ is the meshsize in the $j$-th coordinate direction. For small $h$, this requires a large number of regular grids, all with different cell aspect ratios.

Since there is no distinct finest regular grid in the sparse grid family, one can imagine that the representation of one representative solution is no trivial matter. Without explaining this in detail, we mention that, in principle, there are two ways for obtaining a unique representation of a solution on such a grid family. The first one is based on the hierarchical basis representation $[2,10]$. With this method the solution is distributed over hierarchical components on all available grids and the final representation is obtained by adding all these hierarchical components. Such 
a technique is also widely explored in wavelet theory, and it is almost the natural choice if we consider adaptive grid generation, simply because the hierarchical method is almost adaptive by itself. The second way is by the combination technique $[2,5]$. Here we use standard basis representations on all regular grids, usually the solutions of an ordinary second-order finite-element discretization of the PDE. To obtain a unique solution on the composite grid, we perform an extrapolation, the combination extrapolation, which results in a much more accurate solution than the individual solutions. This technique is the basis for this paper.

The use of the standard bases on the different grids has some advantages. First, we can use existing techniques to discretize and solve the different problems on the individual grids. Secondly, the solutions on the individual grids are completely independent of each other and can be computed in parallel $[3,4,5,7,8]$. In this paper, we apply the combination technique on adaptively generated grids for a two-dimensional problem. The adaptive combination technique has already been studied in $[6,11,12]$, but we impose no other limitations on the data structure than those necessary for the adaptive hierarchical basis technique. In other words we present a technique which has the same flexibility as the hierarchical basis technique, but makes use of the advantages of the combination technique.

To demonstrate the new method we apply it to a model singular perturbation problem [9], which by nature of the problem requires adaptive grids. Special attention is paid to the development of a refinement criterion, as this singular perturbation problem will cause some particular difficulties on sparse grids.

\section{Notation}

Let $\mathbf{k}$ be a multi-integer, $\mathbf{k}=\left(k_{1}, \ldots, k_{d}\right)$, with $k_{i} \in \mathbb{N}_{0}^{+}=\{0,1,2, \ldots\}$ for $i=1, \ldots, d$. We define relational operators between multi-integers by

$$
\mathbf{k} \leq \mathbf{n} \Leftrightarrow\left(k_{1} \leq n_{1} \text { and } k_{2} \leq n_{2} \text { and }, \ldots \text {, and } k_{d} \leq n_{d}\right),
$$

analogously we define $\mathbf{k} \geq \mathbf{n}, \mathbf{k}<\mathbf{n}, \mathbf{k}>\mathbf{n}$ and $\mathbf{k}=\mathbf{n}$. Further we define

$$
\max (\mathbf{k}, \mathbf{n})=\left(\max \left(k_{1}, n_{1}\right), \max \left(k_{2}, n_{2}\right) \cdots \max \left(k_{d}, n_{d}\right)\right)
$$

and $\min (\mathbf{k}, \mathbf{n})$ similarly. The unit vectors $\mathbf{e}_{i}, i=1, \ldots, d$, are defined as $\mathbf{e}_{1}=(1,0, \ldots, 0), \mathbf{e}_{2}=(0,1,0, \ldots, 0), \mathbf{e}_{d}=(0, \ldots, 0,1)$ and we use $\mathbf{e}=(1, \ldots, 1)$. We denote $|\mathbf{k}|=k_{1}+\cdots+k_{d l}$ and $\|\mathbf{k}\|=k_{1}, \ldots, k_{d}$. Further, $\mathbf{k}=(\infty, \ldots, \infty)$ is simply written as $\mathbf{k}=\infty$. Scalar multiplication of multi- integers, for any scalar $c \in \mathbb{R}$, is defined as $c \mathbf{k}=\left(c k_{1}, \ldots, c k_{d}\right)$, and the result of this multiplication is made a multi-integer again, by truncating towards zero, e.g., $\frac{1}{2}(1,2,3,4)=$ $(0,1,1,2)$. Multiplication of multi-integers is defined component-wise, i.e., $\mathbf{k n}=\left(k_{1} n_{1}, \ldots, k_{d} n_{d}\right)$. 


\subsection{Grids}

Let $\Omega \subset \mathbb{R}^{d}$ be (a subset of) a $d$-dimensional rectangular domain with boundary $T$. We assume that $\Omega$ is (a subset of) the union of $S_{1} \times S_{2} \times \cdots \times S_{d}$ unit cubes, for some multi-integer $\mathbf{S}=\left(S_{1}, S_{2}, \ldots, S_{d}\right)$, where $S_{j}$ is the length of $\Omega$ in the $j$-th coordinate direction. We define a regular mesh $\Omega_{\mathrm{k}}$ on $\Omega$, with mesh size $\mathbf{h}_{\mathbf{k}}=2^{-\mathbf{k}}=\left(2^{-k_{1}}, \ldots, 2^{-k_{d}}\right)$. The integer $|\mathbf{k}|=k_{1}+\cdots+k_{d}$ is called the level of grid $\Omega_{\mathbf{k}}$. The coarsest grid is $\Omega_{0}$, with $\mathbf{0}=(0, \ldots, 0)$, and therefore the coarsest level is zero. A cell $\Omega_{\mathrm{kj}}$ is defined by $\left[j_{1} 2^{-k_{1}},\left(j_{1}+1\right) 2^{-k_{1}}\right] \times \cdots \times$ $\left[j_{d} 2^{-k_{d}},\left(j_{d}+1\right) 2^{-k_{d}}\right]$. The grid $\Omega_{\mathbf{k}}$ is a set of cells $\Omega_{\mathbf{k j}}$, defined by $\Omega_{\mathbf{k}}=\left\{\Omega_{\mathbf{k j}} \mid \mathbf{0} \leq \mathbf{j}<\mathbf{S} 2^{\mathbf{k}}, \Omega_{\mathbf{k j}} \subset \Omega\right\}$. The volume of a cell $\Omega_{\mathbf{k j}}, \mathbf{0} \leq \mathbf{j}<\mathbf{S} 2^{\mathbf{k}}$ is denoted by $\left\|\mathbf{h}_{\mathbf{k}}\right\|=h_{k_{1}} \cdots h_{k_{d}}$. The vertices of the grid $\Omega_{\mathbf{k}}$ are denoted by $\Omega_{\mathbf{k}}^{+}$, and $\Omega_{\mathbf{k j}}^{+}$ denotes the $\mathbf{j}$-th vertex on grid $\Omega_{\mathbf{k}}$, with $\mathbf{0} \leq \mathbf{j} \leq \mathbf{S} \mathbf{2}^{\mathbf{k}}$. The vertices $\Omega_{\mathrm{kj}}^{+}$are called dyadic points. The Cartesian coordinates of the dyadic point $\Omega_{\mathrm{kj}}^{+}$are denoted by $\mathbf{x}\left(\Omega_{\mathrm{kj}}^{+}\right)$.

\subsubsection{Ordering of Grids and Cells}

Considering a grid $\Omega_{\mathrm{k}}$ we can refine it in all $d$ directions simultaneously and obtain a sequence of grids $\Omega_{\mathbf{k}+\mathbf{e}}, \Omega_{\mathbf{k}+2 \mathbf{e}}, \ldots$. In this way we get a sequentially ordered family of grids. Actually, such a sequence is the basis for any standard multigrid solution method.

On the other hand, starting with the same grid $\Omega_{\mathrm{k}}$, by refinement we can construct the grids $\Omega_{\mathrm{k}+\mathrm{e}_{\mathrm{i}}}$ for $i=1, \ldots, d$. This process is called semi-refinement. The principle of semi-refinement can be continued recursively for the newly formed refined grids, and thus we obtain an infinite, partially ordered family of grids. We define the virtual family of grids or family of virtual grids as

$$
\begin{aligned}
G_{x} & =\left\{\Omega_{\mathbf{k}} \mid \mathbf{0} \leq k \leq \infty\right\} \\
& \approx\left\{\Omega_{\mathbf{k} \mathbf{j}} \mid \mathbf{0} \leq \mathbf{k} \leq \infty, \quad \mathbf{0} \leq \mathbf{j} \leq \mathbf{S} 2^{\mathbf{k}}\right\} .
\end{aligned}
$$

Since a grid $\Omega_{\mathbf{k}}$ is a set of cells $\left\{\Omega_{\mathbf{k j}} \mid \mathbf{0} \leq \mathbf{j} \leq \mathbf{S} 2^{\mathbf{k}}\right\}$, we also speak of the family of virtual cells. We call these grids and cells virtual because most of them are not found in the actual implementation. For this goal we distinguish between the virtual grids (cells) and the generated grids (cells). Before we give the definition of a generated cell we first define three relations that may exist between cells in the different grids from the family $G_{x}$.

\subsubsection{Relations between Cells}

We define the relations (i) father, (ii) kid, (iii) ancestor between cells in $G_{\infty}$.

Definition 1. Let a direction be denoted by $\mathbf{e}_{i}, i=1, \ldots, d$, and let a cell $\Omega_{\mathbf{k j}} \in G_{\infty}$ be give. Then the father of cell $\Omega_{\mathbf{k j}}$ in the $\mathbf{e}_{i}$-direction is defined by

$$
\mathscr{F}^{\mathbf{e}^{\prime}}\left(\Omega_{\mathbf{k j}}\right)=\Omega_{\mathbf{k}-\mathbf{e}_{i} \cdot \mathbf{j}-\frac{1}{2} \mathbf{j} \mathbf{e}_{t}} .
$$


The existence of a father $\mathscr{F}^{\mathrm{e}_{i}}\left(\Omega_{\mathrm{kj}}\right) \in G_{\infty}$ is not always guaranteed. In fact father $\mathscr{F}^{\mathbf{e}_{i}}\left(\Omega_{\mathbf{k j}}\right)$ does not exist if and only if $k_{i}=0$. In other words, for a cell $\Omega_{\mathbf{k j}}$ all fathers exist except when $\|\mathbf{k}\|=0$.

Definition 2. Let a cell $\Omega_{\mathrm{kj}} \in G_{\infty}$ and a direction $\mathbf{e}_{i}, i=1, \ldots d$, be given. Then the kids of the cell $\Omega_{\mathbf{k j}}$ in the $\mathbf{e}_{i}$-direction, denoted by $\mathscr{K}^{\mathbf{e}_{i}}\left(\Omega_{\mathbf{k j}}\right)$ are defined by

$$
\mathscr{K}^{\mathbf{e}_{i}}\left(\Omega_{\mathbf{k j}}\right)=\left\{\Omega_{\mathbf{k}+\mathbf{e}_{i}, \mathbf{j}+\mathbf{j e} \mathbf{e}_{i}}, \Omega_{\mathbf{k}+\mathbf{e}_{i}, \mathbf{j}+\mathbf{j e}_{i}+\mathbf{e}_{i}}\right\} .
$$

We see that this set of two kinds in the direction $\mathbf{e}_{i}$ always exists in $G_{\infty}$. In the other directions other kids exist, so that in total, in $G_{\infty}$ a cell $\Omega_{\mathbf{k j}}$ has $2 d$ kids in $d$ directions.

Definition 3. Let $\left\{\Omega_{\mathbf{k}_{1} \mathbf{j}_{1}}, \ldots, \Omega_{\mathbf{k}_{p} \mathbf{j}_{p}}\right\}$ be a set of $p$ cells in $G_{\infty}$ with non-empty intersection. Let a dyadic point $\Omega_{\mathrm{ni}}^{+}$be contained in all these $p$ cells. Then the ancestor $\mathscr{A}\left(\Omega_{\mathbf{k}_{1} \mathbf{j}_{1}}, \ldots, \Omega_{\mathbf{k}_{p} \mathbf{j}_{p}}\right) \in G_{\infty}$ is defined as,

$$
\mathscr{A}\left(\Omega_{\mathbf{k}_{\mathbf{l}} \mathbf{j}_{1}}, \ldots, \Omega_{\mathbf{k}_{p} \mathbf{j}_{p}}\right)=\Omega_{\min \left(\mathbf{k}_{1}, \ldots, \mathbf{k}_{p}\right), \mathbf{i}}=\Omega_{\mathbf{n i}}
$$

with $\mathbf{n}=\min \left(\mathbf{k}_{1}, \ldots, \mathbf{k}_{d}\right)$. It is easily verified that $\mathbf{i}$ is uniquely determined. In fact, the ancestor is the finest cell which encloses all cells in $\left\{\Omega_{\mathbf{k}_{1} \mathbf{j}_{1}}, \ldots, \Omega_{\mathbf{k}_{p} \mathbf{j}_{p}}\right\}$. In contrast with the definition of father and kinds, the ancestor does not depend on a direction $\mathbf{e}_{i}$. Notice that, with this definition of an ancestor cell $\mathscr{A}\left(\Omega_{\mathbf{k}_{1} \mathbf{j}_{1}}, \ldots, \Omega_{\mathbf{k}_{p} \mathbf{j}_{p}}\right)$, it possibly coincides with one of its own arguments $\Omega_{\mathbf{k}_{\mathrm{j}} \mathrm{j}_{t}}$, for $t \in\{1, \ldots, p\}$.

\subsubsection{The Generated Grid}

In practice, in order to represent a solution sufficiently accurate and efficient, we want to use as few cells as possible. Therefore, we distinguish between approximation on the virtual family of grids $\mathscr{G}_{\infty}$ and on the much smaller set of generated cells, denoted by $\mathscr{G}$. The set $\mathscr{G}$ is finite, and we denote the number of cells in $\mathscr{G}$ by $|\mathscr{G}|$.

Definition 4. A set of generated cells $\mathscr{G}$, or an adaptive structure $\mathrm{G}$, is a finite subset of $G_{\infty}$ that satisfies the condition

$$
\Omega_{\mathbf{k j}} \in \mathscr{G} \Rightarrow \begin{cases}\text { for all } & i=1, \ldots, d \\ \text { either } & k_{i}=0, \\ \text { or } & \mathscr{F}^{\mathbf{e}_{i}}\left(\Omega_{\mathbf{k j}}\right) \in \mathscr{G} .\end{cases}
$$

In contrast to the virtual set of cells $G_{\infty}$, the possibility exists that, if $\Omega_{k j} \in \mathscr{G}$ this cell has no $\mathrm{e}_{i}$-kids, i.e., $\mathscr{K}^{\mathbf{e}_{i}}\left(\Omega_{\mathrm{kj}}\right) \cap \mathscr{G}=\emptyset$. As a consequence of the definition, if $\left\{\Omega_{\mathbf{k}_{i} \mathbf{j}_{l}} \mid t \in\{1, \ldots, p\}\right\} \in \mathscr{G}$ than also $\mathscr{A}\left(\Omega_{\mathbf{k}_{1} \mathbf{j}_{1}}, \ldots, \Omega_{\mathbf{k}_{p} \mathbf{j}_{p}}\right) \in \mathscr{G}$, due to the existence of all $d$ fathers of every cell $\Omega_{\mathrm{k}_{t} j_{t}}, t \in\{1, \ldots, p\}$. The notion of set of generated cells is comparable with the so called active indices in [6]. Corresponding with a set of 
generated cells $\mathrm{G}$ we can also construct a composite grid by considering all points $\Omega_{\mathbf{k j}}^{+}$corresponding with an arbitrary set of generated cells $\mathrm{G}$. This is called the adaptive sparse grid $\mathscr{G}^{+}$.

The set $\mathscr{G}_{l}^{+}$, defined by

$$
\mathscr{G}_{l}^{+}=\left\{\Omega_{\mathrm{kj}}^{+} \mid \Omega_{\mathrm{kj}}^{+} \subset \bar{\Omega}_{\mathrm{kj}}, \Omega_{\mathrm{kj}}^{+} \in \mathscr{G}_{\ell}\right\}
$$

is called the adaptive sparse grid on level $l$.

\subsubsection{The Sparse Family of Grids}

A special case of generated grids is formed if we consider all cells $\left\{\Omega_{\mathbf{k j}}\right\}$, with $|\mathbf{k}|$ bounded by some level $\ell$. This set of grids or set of cells is defined as the sparse family of grids and is denoted by

$$
\begin{aligned}
\mathscr{G}_{\ell} & =\left\{\Omega_{\mathbf{k}}|| \mathbf{k} \mid \leq \ell\right\}, \\
& \approx\left\{\Omega_{\mathbf{k} \mathbf{j}}|\mathbf{k}| \leq \ell, \mathbf{0} \leq \mathbf{j}<\mathbf{S} 2^{\mathbf{k}}\right\}, \quad \Omega_{\mathbf{k j}} \subset \Omega .
\end{aligned}
$$

The sparse grid is obtained by constructing the composite grid, i.e., considering the union of grid points $\left\{\Omega_{\mathbf{k j}}^{+}|| \mathbf{k} \mid \leq \ell, \mathbf{0} \leq j \leq \mathbf{S} 2^{\mathbf{k}}\right\}$.

\subsection{Bases and Spaces}

\subsubsection{Standard Representation}

An approximation of a function $u \in \mathscr{C}(\Omega)$ on $\Omega_{\mathrm{k}} \in G_{\infty}$ can be given by

$$
u \approx u_{\mathrm{k}}=\sum_{\mathbf{j}} u_{\mathrm{kj}} \varphi_{\mathrm{kj}}
$$

with $u_{\mathbf{k j}}=u_{\mathbf{k}}\left(\mathbf{x}\left(\Omega_{\mathbf{k j}}^{+}\right)\right)$and $\varphi_{\mathbf{k j}}$ the usual piecewise $d$-linear basis function with $\operatorname{supp}\left(\varphi_{\mathbf{k j}}\right)=\bigcup_{\mathbf{j} \leq \mathbf{m} \leq \mathbf{j}+\mathbf{e}} \Omega_{\mathbf{k m}} \cap \Omega$, of tensor product type. With $u(x)=u_{\mathbf{k}}(x)$ for all $x \in \Omega_{\mathbf{k}}^{+}$, this $u_{\mathbf{k}}$ is called the standard representation of $u$ on $\Omega_{\mathbf{k}}$. For any $\mathbf{k} \geq \mathbf{0}$ and for all piecewise $d$-linear approximations of $\mathscr{C}(\Omega)$-functions, the standard basis $B_{\mathbf{k}}$ is defined as

$$
B_{\mathbf{k}}=\left\{\varphi_{\mathbf{k j}} \mid \mathbf{0} \leq j \leq \mathbf{S} 2^{\mathbf{k}}\right\}
$$

and the corresponding space of approximating piecewise $d$-linear functions is

$$
V_{\mathbf{k}}=\operatorname{Span}\left(B_{\mathbf{k}}\right)
$$

Further, for $\ell \in \mathbb{N}_{0}^{+}$we define,

$$
B_{\ell}=\left\{B_{\mathbf{k}}|| \mathbf{k} \mid=\ell\right\}
$$

and the corresponding function space is 


$$
V_{\ell}=\operatorname{Span}\left(B_{\ell}\right)
$$

In a natural way the set of coefficients $\left\{u_{\mathbf{k j}}\right\}_{\mathbf{0} \leq \mathbf{j} \leq \mathbf{S} 2^{k}}$ in (2) is an element in a vector space $\mathbf{V}_{\mathbf{k}}$. By concatenation of such vectors we define the vector space $\mathbf{V}_{\ell}=\otimes_{|\mathbf{k}|=\ell} \mathbf{V}_{\mathrm{k}}$. The vector space $\overline{\mathbf{V}}_{\ell}$ is defined by $\overline{\mathbf{V}}_{\ell}=\otimes_{j=0}^{\ell} \mathbf{V}_{j}$. If no confusion is possible we use the notation $u_{\mathbf{k}}$ both for a grid function $u_{\mathbf{k}} \in V_{\mathbf{k}}$ and for a vector with coefficients for the grid function $u_{\mathbf{k}} \in \mathbf{V}_{\mathbf{k}}$. Notice that we can extend this vector space to the representation $u_{\ell}=\otimes_{|\mathbf{k}|=\ell} u_{\mathbf{k}}$.

The basis $B_{\ell}$ is a basis for the approximations that can be represented on $\mathscr{G}_{\ell}$, and similar $V_{\ell}$ is a function space spanned on $\mathscr{G}_{\ell}$. If we consider an adaptive structure $\mathscr{G}$ we can define bases, function spaces and vector spaces in the same way. By $B_{\mathscr{G}}$, $V_{\mathscr{G}}$ and $\overline{\mathbf{V}}_{\mathscr{G}}$ we denote respectively the notions for an adaptive structure corresponding with $B_{\ell}, V_{\ell}$ and $\overline{\mathbf{V}}_{\ell}$.

\subsubsection{Hierarchical Representation}

A point $\Omega_{\mathrm{kj}}^{+}$, is called a hierarchical point if it does not appear on a coarser grid, i.e., $\mathbf{x}\left(\Omega_{\mathbf{k j}}^{+}\right) \notin\left\{\mathbf{x}\left(\Omega_{\mathrm{ni}}^{+}\right) \mid \mathbf{n} \leq \mathbf{k}, \mathbf{n} \neq \mathbf{k}, \mathbf{0} \leq \mathbf{i} \leq \mathbf{S} 2^{\mathbf{n}}\right\}$. It is easily shown that hierarchical points $\Omega_{\mathrm{kj}}^{+}, \mathbf{k} \neq \mathbf{0}$, satisfy $\prod_{i=1, k_{i} \neq 0}^{d} \dot{j}_{i}$ is odd. Therefore we denote the hierarchical points in short by ' $\Omega_{\mathbf{k j}}^{+}, \mathbf{j}$ odd'.

We see that basis function $\varphi_{\mathrm{kj}}$, corresponding with hierarchical points $\Omega_{\mathrm{kj}}^{+}$make bases for $V_{\mathbf{k}}$ and $V_{\ell}$.

The hierarchical basis $\tilde{B}_{\mathbf{k}}$ for $V_{\mathbf{k}}$ is now defined by

$$
\tilde{B}_{\mathbf{k}}=\left\{\varphi_{\mathbf{n j}} \mid \varphi_{\mathbf{n j}} \in B_{\mathbf{n}}, \mathbf{n} \leq \mathbf{k}, \quad \mathbf{j} \text { odd }\right\}
$$

and the hierarchical basis for $V_{\ell}$ by $\Omega_{\mathbf{k}} \in \mathscr{G}_{\ell}$ is,

$$
\tilde{B}_{\ell}=\left\{\varphi_{\mathbf{n} \mathbf{j}}\left|\varphi_{\mathbf{n} \mathbf{j}} \in B_{\mathbf{n}},\right| \mathbf{n} \mid \leq \ell, \quad \mathbf{j} \text { odd }\right\} .
$$

The piecewise $d$-linear approximations of functions using the bases $\tilde{B}_{\mathbf{k}}$ and $\tilde{B}_{\ell}$ and written as

$$
\begin{aligned}
& \tilde{u}_{\mathbf{k}}=\sum_{\mathbf{n} \leq \mathbf{k}} \sum_{\mathbf{j} \text { odd }} \tilde{u}_{\mathbf{n j}} \varphi_{\mathbf{n j}}, \\
& \tilde{u}_{\ell}=\sum_{0 \leq|\mathbf{k}| \leq \ell} \sum_{\mathbf{j} \text { odd }} \tilde{u}_{\mathbf{k j}} \varphi_{\mathbf{k j}} .
\end{aligned}
$$

Notice the complete difference between (2) and (3). We use the same notation $\tilde{u}_{\ell}$ to denote $\tilde{u}_{\ell} \in \overline{\mathbf{V}}_{\ell}$, i.e., a vector with zeroes in the positions corresponding with nonhierarchical points. Further we write $\tilde{u}_{\uparrow \ell \ell-1}=\sum_{|\mathbf{k}|=\ell} \sum_{\mathbf{j}} \tilde{u}_{\mathbf{k j}} \varphi_{\mathbf{k j}}$, to identify the hierarchical contribution from level $\ell$. 


\subsection{Operators}

Let $\mathbf{m} \geq \mathbf{k}$, then the piecewise linear prolongation operator $P_{\mathbf{m k}}: V_{\mathbf{k}} \rightarrow V_{\mathbf{m}}$, for interpolation of a function $u_{\mathrm{k}}$ on the grid $\Omega_{\mathrm{k}}$ to a function $u_{\mathrm{m}}$ on the grid $\Omega_{\mathrm{m}}$ is defined by

$$
P_{\mathbf{m k}}\left(u_{\mathbf{k}}\right)=u_{\mathbf{m}}, \quad \text { with } u_{\mathbf{m}}(x)=u_{\mathbf{k}}(x), \quad \forall x \in \Omega,
$$

and we define the piecewise linear restriction $R_{\mathbf{k m}}: V_{\mathbf{m}} \rightarrow V_{\mathbf{k}}$ as

$$
R_{\mathbf{k m}}\left(u_{\mathbf{m}}\right)=u_{\mathbf{k}}, \quad \text { with } u_{\mathbf{k}}(x)=u_{\mathbf{m}}(x), \quad \forall x \in \Omega_{\mathbf{k}}^{+} .
$$

If $\mathbf{m}=\min (\mathbf{k}, \mathbf{n})$, for arbitrary $\mathbf{k}$ and $\mathbf{n}$, the projection operator $\Pi_{\mathbf{k} \mathbf{n}}: V_{\mathbf{n}} \rightarrow V_{\mathbf{k}}$ is defined by

$$
\Pi_{\mathrm{kn}}\left(u_{\mathrm{n}}\right)=P_{\mathrm{km}} R_{\mathrm{mn}}\left(u_{\mathrm{n}}\right)
$$

with $P_{\mathrm{mk}}, R_{\mathrm{nm}}$ the above piecewise linear prolongation and restriction operators. This operator $\Pi_{\mathrm{kn}}$ projects the function $u_{\mathrm{n}}$ of grid $\Omega_{\mathrm{n}}$ onto grid $\Omega_{\mathrm{k}}$. Notice that in a partially ordered set of grids, the minimum $\mathbf{m}$ is not necessarily equal to $\mathbf{k}$ or $\mathbf{n}^{1}$. We use the symbol $\Pi_{\mathbf{k n}}$ with the understanding that $\Pi_{\mathbf{k n}}=0$ if $\min \left(k_{i}, n_{i}\right)<0$ for any $i-1, \ldots, d$. A treatment of some properties of these operators is given in [10].

\section{The Adaptive Combination Formula}

The combination technique for sparse grids makes use of the standard basis $B_{\mathbf{n}}$, for all generated grids $\Omega_{n} \in \mathscr{G}_{l}$. Every grid has its own discretization and the discrete problems on the different grids $\Omega_{\mathrm{n}} \in \mathscr{G}_{\ell}$ are solved separately, possibly in parallel $[3-5,7,8]$. Once the solution process on the different grids is completed one composes a final unique combination solution on (a part of) a virtual grid $\Omega_{\mathbf{k}}$. We define the $d$-dimensional combination operator $C_{\mathrm{k}}^{d}: V_{\ell} \rightarrow V_{\mathbf{k}}$ or the $d$-dimensional combination formula for the extrapolation on the virtual grid $\Omega_{\mathbf{k}}$ by

$$
u_{\mathbf{k}}=C_{\mathbf{k}}^{d}\left(u_{\ell}\right)=\sum_{c=0}^{d-1}(-1)^{c}\left(\begin{array}{c}
d-1 \\
c
\end{array}\right) \sum_{|\mathbf{n}|=\ell-c} \Pi_{\mathbf{k n}}\left(u_{\mathbf{n}}\right) .
$$

This combination formula is introduced for the case of "complete sparse grids", $\mathscr{G}_{\ell}$ by [2]. For an adaptive generated set of cells $\mathscr{G}$, we cannot use this extrapolation formula. Therefore, in this section we develop a combination formula for an arbitrary adaptive set of generated cells $\mathscr{G}$.

The adaptive combination technique has already been considered in $[6,11,12]$. The authors, however, impose more restrictions to the adaptive structure $\mathscr{G}$ than mentioned for an adaptive structure in Definition 4. The adaptive combination ${ }^{1}$ The value at a particular point $\Omega_{\mathrm{kj}}^{+}$for these operators is denoted by $\left(R_{\mathrm{km}}\left(u_{\mathrm{m}}\right)\right)_{\mathbf{j}},\left(P_{\mathrm{kn}}\left(u_{\mathrm{n}}\right)\right)_{\mathbf{j}}$, and
$\left(\Pi_{\mathrm{kn}}\left(u_{\mathrm{n}}\right)\right)_{\mathbf{j}}$, respectively. 
formula presented here does not impose additional requirements on the structure of $\mathscr{G}$. Moreover, during the generation of $\mathscr{G}$ we do not need to keep any administration with regards to the structure of $\mathscr{G}$. So the creation of an adaptive combination solution is completely decoupled from the generation of $\mathscr{G}$.

\subsection{Regular Combination Formula}

We first introduce concepts needed to the derivation of the adaptive combination formula, and then we apply these concepts for the derivation of the regular and adaptive combination formulae. For ease of notation, from this point on we describe only the two-dimensional case.

Consistent approximations: Two approximations, $u_{\mathbf{k}}$ on $\Omega_{\mathbf{k}} \in G_{\infty}$ and $u_{\mathbf{n}}$ on $\Omega_{\mathbf{n}} \in G_{\infty}$, are called consistent [10], if for grid $\Omega_{\mathbf{m}} \in G_{\infty}$, with $\mathbf{m}=\min (\mathbf{k}, \mathbf{n})$ we have,

$$
\Pi_{\mathrm{mk}}\left(u_{\mathrm{k}}\right)=\Pi_{\mathrm{mn}}\left(u_{\mathrm{n}}\right) .
$$

The approximations are called consistent at a point $\Omega_{\mathrm{mj}}^{+}$if

$$
\left(\Pi_{\mathrm{mk}}\left(u_{\mathrm{k}}\right)\right)_{\mathbf{j}}=\left(\Pi_{\mathrm{mn}}\left(u_{\mathrm{n}}\right)\right)_{\mathbf{j}}
$$

Extrapolation: Let us consider the grids $\left\{\Omega_{\mathbf{k}-\mathbf{e}_{1}}, \Omega_{\mathbf{k}-\mathbf{e}_{2}}, \Omega_{\mathbf{k}-\mathbf{e}}\right\} \subset \mathscr{G}_{\ell}$, with their approximations $u_{\mathbf{k}-\mathbf{e}_{1}}, u_{\mathbf{k}-\mathbf{e}_{2}}, u_{\mathbf{k}-\mathbf{e}}$. The two-dimensional extrapolated approximation for the grid $\Omega_{\mathbf{k}} \subset G_{\infty}$ is defined by

$$
u_{\mathbf{k}}=\mathscr{E}\left(u_{\mathbf{k}-\mathrm{e}_{1}}, u_{\mathbf{k}-\mathbf{e}_{2}}, u_{\mathbf{k}-\mathbf{e}}\right)=\prod_{\mathbf{k}, \mathbf{k}-\mathbf{e}_{1}} u_{\mathbf{k}-\mathbf{e}_{1}}+\prod_{\mathbf{k}, \mathbf{k}-\mathbf{e}_{2}} u_{\mathbf{k}-\mathbf{e}_{2}}-\prod_{\mathbf{k}, \mathbf{k}-\mathrm{e}} u_{\mathbf{k}-\mathbf{e}} .
$$

The local extrapolated approximation on the grid $\mathbf{k}$ at the point $\Omega_{\mathbf{k j}}^{+} \in \mathscr{G}_{\infty}$ is defined by

$$
u_{\mathbf{k j}}=\left(\Pi_{\mathbf{k}, \mathbf{k}-\mathbf{e}_{1}} u_{\mathbf{k}-\mathbf{e}_{1}}\right)_{\mathbf{j}}+\left(\Pi_{\mathbf{k}, \mathbf{k}-\mathbf{e}_{2}} u_{\mathbf{k}-\mathbf{e}_{2}}\right)_{\mathbf{j}}-\left(\Pi_{\mathbf{k}, \mathbf{k}-\mathbf{e}} u_{\mathbf{k}-\mathrm{e}}\right)_{\mathbf{j}}
$$

The extrapolated approximation and the local extrapolated approximation are both defined for $\Omega_{\mathbf{k}} \in G_{x}$, whereas the original approximations on the grids $\left\{\Omega_{\mathbf{k}-\mathbf{e}_{1}}, \Omega_{\mathbf{k}-\mathbf{e}_{2}}, \Omega_{\mathbf{k}-\mathbf{e}}\right\}$ are defined on $\mathscr{G}$. In particular, for a given $\mathscr{G}=\mathscr{G}_{l}$, extrapolation to a virtual grid occurs when $|\mathbf{k}|=\ell+1$.

The hierarchical surplus: Let us consider the consistent approximations $u_{\mathbf{k}}, u_{\mathbf{k}-\mathbf{e}_{1}}, u_{\mathbf{k}-\mathbf{e}_{2}}, u_{\mathbf{k}-\mathbf{e}}$ on the grids $\left\{\Omega_{\mathbf{k}}, \Omega_{\mathbf{k}-\mathbf{e}_{1}}, \Omega_{\mathbf{k}-\mathbf{e}_{2}}, \Omega_{\mathbf{k}-\mathbf{e}}\right\} \subset \mathscr{G}_{\ell}$. The hierarchical surplus $\tilde{u}_{\mathrm{k}}$ for the grid $\Omega_{\mathrm{k}} \subset \mathscr{G}_{\ell}$ is defined as

$$
\tilde{u}_{\mathbf{k}}=u_{\mathbf{k}}-\Pi_{\mathbf{k}, \mathbf{k}-\mathrm{e}_{1}} u_{\mathbf{k}-\mathrm{e}_{1}}-\Pi_{\mathbf{k}, \mathbf{k}-\mathrm{e}_{2}} u_{\mathbf{k}-\mathrm{e}_{2}}+\Pi_{\mathbf{k}, \mathbf{k}-\mathbf{e}} u_{\mathbf{k}-\mathbf{e}}
$$

The local hierarchical surplus [10] in the dyadic point $\Omega_{\mathbf{k j}}^{+}$is calculated by

$$
\tilde{u}_{\mathrm{kj}}=u_{\mathrm{kj}}-\left(\Pi_{\mathrm{k}, \mathbf{k}-\mathrm{e}_{1}} u_{\mathbf{k}-\mathrm{e}_{1}}\right)_{\mathbf{j}}-\left(\Pi_{\mathbf{k}, \mathbf{k}-\mathrm{e}_{2}} u_{\mathbf{k}-\mathrm{e}_{2}}\right)_{\mathbf{j}}+\left(\Pi_{\mathbf{k}, \mathbf{k}-\mathrm{e}} u_{\mathbf{k}-\mathrm{e}}\right)_{\mathbf{j}}
$$


In contrast to the hierarchical surplus $\tilde{u}_{\mathbf{k}}$, which is only defined for approximations $\tilde{u}_{\mathbf{k}} \subset \mathscr{G}_{\ell}$, the local hierarchical surplus is defined for all consistent approximations on $\mathscr{G}$.

With these concepts we show that the regular combination technique gives the same result as the hierarchical basis representation of a consistent solution. This statement is the subject of the following lemma.

Lemma 1. Let all approximations $u_{\mathbf{k}} \subset u_{\ell}$ on the grids $\Omega_{\mathbf{k}} \in \mathscr{G}_{\ell}$ be consistent with respect to each other. The combination formula (8) with $d=2$, for the extrapolation to the grid $\Omega_{\infty}$ results in the same approximation as the hierarchical representation, $u_{\infty}=\sum_{\mathbf{n}<\infty} \sum_{\mathbf{j}} \tilde{u}_{\mathbf{n j}} \varphi_{\mathbf{n j}}$

$$
\sum_{0 \leq|\mathbf{n}| \leq \ell} \tilde{u}_{\mathbf{n}}=C_{\infty}^{2}\left(u_{\ell}\right)
$$

Proof: By calculation of the hierarchical surplus, Eq. (11), we can derive

$$
\sum_{|\mathbf{n}|=\ell} \tilde{u}_{\mathbf{n}}=C_{\infty}^{2}\left(u_{\ell}\right)-C_{\infty}^{2}\left(u_{\ell-1}\right)
$$

further, by definition $C_{\infty}^{2}\left(u_{0}\right)=u_{0}=\tilde{u}_{0}$. So, by recursion,

$$
\begin{aligned}
C_{\infty}^{2}\left(u_{\ell}\right) & =\sum_{|\mathbf{n}|=\ell} \tilde{u}_{\mathbf{n}}+C_{\infty}^{2}\left(u_{\ell-1}\right) \\
& =\sum_{|\mathbf{n}|=\ell} \tilde{u}_{\mathbf{n}}+\sum_{|\mathbf{n}|=\ell-1} \tilde{u}_{\mathbf{n}}+\cdots+\sum_{|\mathbf{n}|=0} \tilde{u}_{\mathbf{n}} \\
& =\sum_{0 \leq|\mathbf{n}| \leq \ell} \tilde{u}_{\mathbf{n}} .
\end{aligned}
$$

An important assumption in the above Lemma 1 is the fact that we considered consistent approximations. However, by recursive application the combination formula can also be applied to the virtual grids $\Omega_{\mathbf{k}} \subset G_{\infty}$. Extrapolation to all grids on level $\ell+1$ and subsequently to the levels $\ell+2, \ldots, 2 \ell$, will yield the same approximation. However, in the latter case it is not strictly necessary that the solutions are consistent, since consistency of the solutions was no requirement for the extrapolation equation (9). Therefore we are allowed to use solutions which are all affected by their own discretization error, we say the solutions are consistent up to discretization error. Notice that the accuracy in the combination solution as described in [13] is obtained by cancellation of these discretization errors on the different grids, assumed that an expansion on the discretization error exists of the type (for $d=2$ ) $u_{h_{1}, h_{2}}=u^{*}+e x\left[h_{1}\right]+e y\left[h_{2}\right]+r\left[h_{1} h_{2}\right]$. (i.e., the error is the sum of three parts, where e.g. ex $\left[h_{1}\right]$ depends only on $x, y$ and $h_{1}$.) 


\subsection{Two-Dimensional Adaptive Combination Formula}

If the generated grids are not $\mathscr{G}_{\ell}$ but an arbitrary set of generated cells $\mathscr{G}$, we cannot use the combination formula (8). In the case when the approximations are no longer defined on the entire domain $\Omega$ but only on a couple of cells $\Omega_{\mathbf{k j}} \subset \mathscr{G}$ in the grid $\Omega_{\mathbf{k}} \subset G_{\infty}$, to still obtain a combination solution, we can extend the set of cells $\mathscr{G}$ by virtual cells from $G_{\infty}$ and use the local extrapolation (10) to "fill up" the solutions in the additional cells of $G_{\infty}$. In practice, to save an essential amount of computational work, we do not want to create such additional cells. Therefore, to arrive at the same goal, we introduce the adaptive combination formula.

In order to introduce the adaptive combination formula, we first give two definitions for particular sets of cells.

Definition 5. Given an adaptive structure $\mathscr{G}$, the set of finest cells for a dyadic point $\Omega_{\mathbf{k j}}^{+} \in \bar{\Omega}_{j} \in G_{\infty}$ in a direction $\mathbf{e}_{i}$ is denoted by ${ }^{0} \operatorname{pos}_{\mathbf{k j}}^{\mathbf{e}_{i}}$, and defined by,

$$
{ }^{0} \mathscr{P}_{\mathrm{kj}}^{\mathbf{e}_{i}}=\left\{\Omega_{\mathrm{ns}} \mid \mathscr{K}^{\mathbf{e}_{i}}\left(\Omega_{\mathrm{ns}}\right) \cap \mathscr{G}=\emptyset, \Omega_{\mathrm{kj}}^{+} \in \bar{\Omega}_{\mathrm{ns}}\right\}
$$

Definition 6. Given an adaptive structure $\mathscr{G}$, the set of (first degree) ancestors for a dyadic point $\Omega_{\mathrm{kj}}^{+} \in \bar{\Omega}_{\mathrm{mr}} \in G_{\infty}$ in a direction $\mathbf{e}_{i}$ is denoted by ${ }^{1} \mathscr{p}_{\mathbf{k j}}^{\mathbf{e}_{i}}$, and defined by

$$
\begin{array}{r}
{ }^{1} \mathscr{P}_{\mathbf{k j}}^{\mathbf{e}_{i}}=\left\{\Omega_{\mathbf{m r}}=\mathscr{A}\left(\Omega_{\mathbf{k p}}, \Omega_{\mathbf{n q}}\right) \mid\left\{\Omega_{\mathbf{k p}}, \Omega_{\mathbf{n q}}\right\} \subset{ }^{0} \mathscr{P}_{\mathbf{k j}}^{\mathbf{e}_{i}},\right. \\
\left.\mathbf{n}=\mathbf{k}+\mathbf{e}_{t}, t \neq i, \Omega_{\mathbf{k j}}^{+} \in \bar{\Omega}_{\mathbf{m r}}\right\} .
\end{array}
$$

To illustrate the definition of the set of finest cells and the set of ancestors we give an example in Fig. 1. In the figure one should not consider the squares as the representation of a regular grid. In the figure the squares represent cells which all contain a given (arbitrary) dyadic point $\Omega_{\mathrm{kj}}^{+}$. The dashed ones are virtual cells. In the example the direction chosen is $\mathbf{e}_{2}$. This means that one has to search in "columns" for the finest cells. To get an ancestor cell, one has to look in the two "neighbor columns" for their finest cells and then the ancestor is defined as the minimum cell with respect to the finest cells.

One observes that it is possible that a particular cell is part of ${ }^{0} \mathscr{P}_{\mathbf{k j}}^{\mathbf{e}_{i}}$ and part of ${ }^{1} \mathscr{P}_{\mathbf{k j}}^{\mathbf{e}_{i}}$ as well.

The following definition describes the adaptive combination formula

Definition 7. Consider a given set of generated cells, $\mathscr{G}$, a dyadic point $\Omega_{\mathrm{kj}}^{+} \in G_{\infty}$ and a direction $\mathbf{e}_{i}$. Then the adaptive combination formula for the point $\Omega_{\mathbf{k j}}^{+}$reads

$$
u_{\mathbf{k j}}=\sum_{\Omega_{\mathbf{n s}} \in \in^{0}, \mathcal{P}_{\mathrm{kj}}^{\mathrm{e}_{\mathrm{j}}}}\left(\Pi_{\mathbf{k n}}\left(u_{\mathbf{n s}}\right)\right)_{\mathbf{j}}-\sum_{\Omega_{\mathbf{n s}} \in \in^{1}, \mathcal{P}_{\mathrm{kj}}^{\mathrm{e}_{\mathrm{j}}}}\left(\Pi_{\mathbf{k n}}\left(u_{\mathbf{n s}}\right)\right)_{\mathbf{j}} .
$$

Lemma 2. In the case $\mathscr{G}=\mathscr{G}_{\ell}, u_{\mathbf{k}}$ in the adaptive combination formula gives the same result as (8) with $d=2$. 


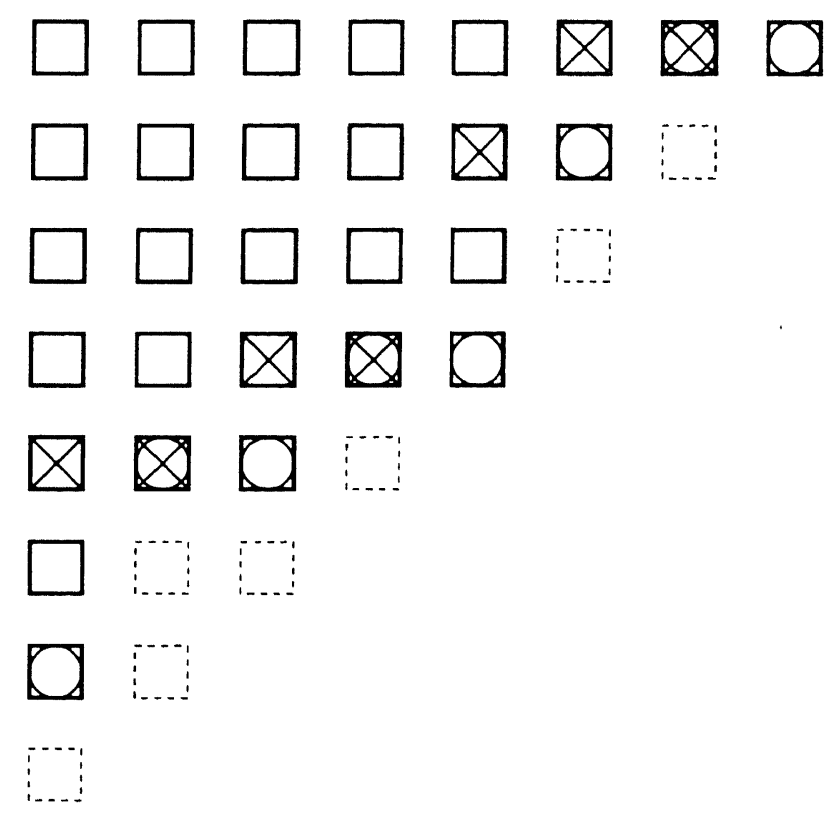

Figure 1. Example of the sets ${ }^{0} \mathscr{P}_{\mathrm{kj}}^{\mathrm{e}_{t}}$ (circles) and ${ }^{1} \mathscr{P}_{\mathrm{kj}}^{\mathrm{e}_{t}}$ (crosses)

Proof: The proof is immediate if we see that in this case ${ }^{0} \mathscr{P}_{\mathrm{kj}}^{\mathrm{e}_{i}}=\mathscr{G}_{\ell} \backslash \mathscr{G}_{\ell-1}$ and ${ }^{1} \mathscr{P}_{\mathrm{kj}}^{\mathrm{e}_{i}}=\mathscr{G}_{\ell-1} \backslash \mathscr{G}_{\ell-2}$.

Remark. In the definition of the sets ${ }^{0} \mathcal{P}_{\mathbf{k j}}^{\mathbf{e}_{i}}$ and ${ }^{1} \mathfrak{p}_{\mathbf{k j}}^{\mathbf{e}_{i}}$, we use a direction $\mathbf{e}_{i}$. Depending on the adaptive structure $\mathscr{G}$, generally, for different directions $\mathbf{e}_{j} \neq \mathbf{e}_{i}$ we will generate different sets ${ }^{0}, \boldsymbol{p}_{\mathbf{k j}}^{\mathbf{e}_{i}}$ and ${ }^{1}, \boldsymbol{p}_{\mathbf{k j}}^{\mathbf{e}_{i}}$. This observation leads to the following lemma.

Lemma 3. The adaptive combination formula introduced in Definition 7 is invariant in the direction $\mathbf{e}_{i}$.

Proof: We already saw that, for a given $\mathbf{e}_{i}$-direction, possible ${ }^{0} \mathcal{P}_{\mathbf{k j}}^{\mathbf{e}_{t}} \cap{ }^{1}: \mathcal{P}_{\mathbf{k j}}^{\mathbf{e}_{i}}=2^{\mathbf{e}_{i j}} \neq \emptyset$. This implies that the corresponding terms in (15) cancel. This observation leads to the proof of the lemma by showing that the sets ${ }^{0}, \mathfrak{p}_{\mathbf{k j}}^{\mathbf{e}_{i}} \backslash \mathcal{Z}_{\mathrm{kj}}^{\mathbf{e}_{i}}$ and ${ }^{1}, \mathcal{P}_{\mathbf{k j}}^{\mathbf{e}_{i}} \backslash \mathscr{2}_{\mathbf{k j}}^{\mathbf{e}_{i}}$ are independent of the $\mathbf{e}_{i}$-direction.

We see that ${ }^{0} \mathscr{P}_{\mathbf{k j}}^{\mathbf{e}_{i}} \backslash \mathcal{Z}_{\mathrm{kj}}^{\mathbf{e}_{i}}$ is the set of cells that are the finest cells in the $\mathbf{e}_{i}$-direction and not ancestors in the other direction. This implies that this set is the collection of cells that are finest (i.e., have no kids) in all directions.

On the other hand we see that ${ }^{1} \mathscr{P}_{\mathbf{k j}}^{\mathbf{e}_{i}} \mathfrak{2}_{\mathbf{k j}}^{\mathbf{e}_{1}}$ is the set of cells that are first degree ancestor in the $\mathbf{e}_{j}$-direction and not a finest cell in the $\mathbf{e}_{i}$-direction. This means that such a cell has kids in all directions but lacks grandchildren in the $\mathbf{e}_{i}-\mathbf{e}_{j}$-direction. 
Thus we see that the sets ${ }^{0} \mathscr{P}_{\mathbf{k j}}^{\mathbf{e}_{i}} \backslash \mathscr{Q}_{\mathbf{k j}}^{\mathbf{e}_{i}}$ and ${ }^{1} \mathscr{P}_{\mathbf{k j}}^{\mathbf{e}_{i}} \backslash \mathscr{Q}_{\mathbf{k j}}^{\mathbf{e}_{i}}$ are independent of the direction $\mathbf{e}_{j}$, and, therefore, that $u_{\mathrm{kj}}$ in (15) is independent of the direction $\mathbf{e}_{i}$.

Remark. We can characterize the non-cancelling sets in the above proof in direction independent notation as

$$
\begin{aligned}
{ }^{0} \mathscr{P}_{\mathbf{k j}}^{\mathbf{e}_{i}} \backslash \mathscr{Q}_{\mathbf{k j}}^{\mathbf{e}_{i}} & =\left\{\Omega_{\mathrm{mr}} \mid \mathscr{K}^{\mathbf{e}_{i}}\left(\Omega_{\mathrm{mr}}\right) \cap \mathscr{G}=\emptyset, \Omega_{\mathrm{kj}}^{+} \in \Omega_{\mathrm{mr}}\right\}, \\
{ }^{1} \mathscr{P}_{\mathbf{k j}}^{\mathbf{e}_{i}} \backslash \mathscr{Q}_{\mathbf{k j}}^{\mathbf{e}_{i}} & =\left\{\Omega_{\mathrm{mr}} \mid \mathscr{K}^{\mathbf{e}_{i}}\left(\Omega_{\mathrm{mr}}\right) \cap \mathscr{G} \neq \emptyset, \mathscr{K}^{\mathbf{e}_{i}}\left(\mathscr{K}^{\mathbf{e}_{i}}\left(\Omega_{\mathrm{mr}}\right)\right) \cap \mathscr{G}=\emptyset, 1=1,2\right\} .
\end{aligned}
$$

This insight yields the way to generalize formula (15) to higher dimensions.

In contrast with the standard combination formula, equation (8), where we have the same formula for each dyadic grid point $\Omega_{\mathrm{kj}}^{+}$of the virtual grid, for the adaptive combination formula we have generally a different formula for every dyadic point $\Omega_{\mathbf{k j}}^{+}$of the virtual grid. This means that for each dyadic point $\Omega_{\mathbf{k j}}^{+}$we need to find the new sets ${ }^{0} \mathscr{P}_{\mathrm{kj}}^{\mathbf{e}_{t}}$ and ${ }^{1} \mathscr{P}_{\mathbf{k j}}^{\mathbf{e}_{t}}$. So, the adaptive combination formula is a point-wise formula. With a proper data-structure, however, the administration takes a negligible amount of work.

With the above insight we easily prove, similar to Lemma 1, that for consistent approximations the adaptive combination formula (15) results in the same approximation on the virtual grid as the hierarchical decomposition.

\section{Refinement Strategy}

In $[1,6,11]$ adaptively refined sparse grids are used together with the hierarchical decomposition of the solution. Mostly the authors consider the hierarchical corrections as a natural threshold for the refinement strategy. An additional advantage of the use of hierarchical representations is the fact that one does not need to calculate internal boundary conditions. This is because two hierarchical basis functions on the same grid do not share a common support. If we use standard basis representations on the different cells $\Omega_{\mathrm{kj}} \subset \mathscr{G}$, we do not have these particular advantages that go with the hierarchical basis representation.

Before we describe our actual refinement criterion, we discuss some particular difficulties that may appear with non-smooth solutions. In particular, such remarks are relevant for the numerical singular perturbation problem that we will study in some detail in Section 5.

\subsection{Steep Gradients}

In Fig. 2 we give a simple example of a one-dimensional solution which contains a steep gradient. The figure shows the hierarchical decomposition together with the standard basis representation for the different levels. We make two observations related to the use of the hierarchical correction $\tilde{u}_{\mathrm{kj}}$ as a criterion for refinement, say we stop refinement if $\tilde{u}_{\mathrm{kj}} \leq \varepsilon$. 


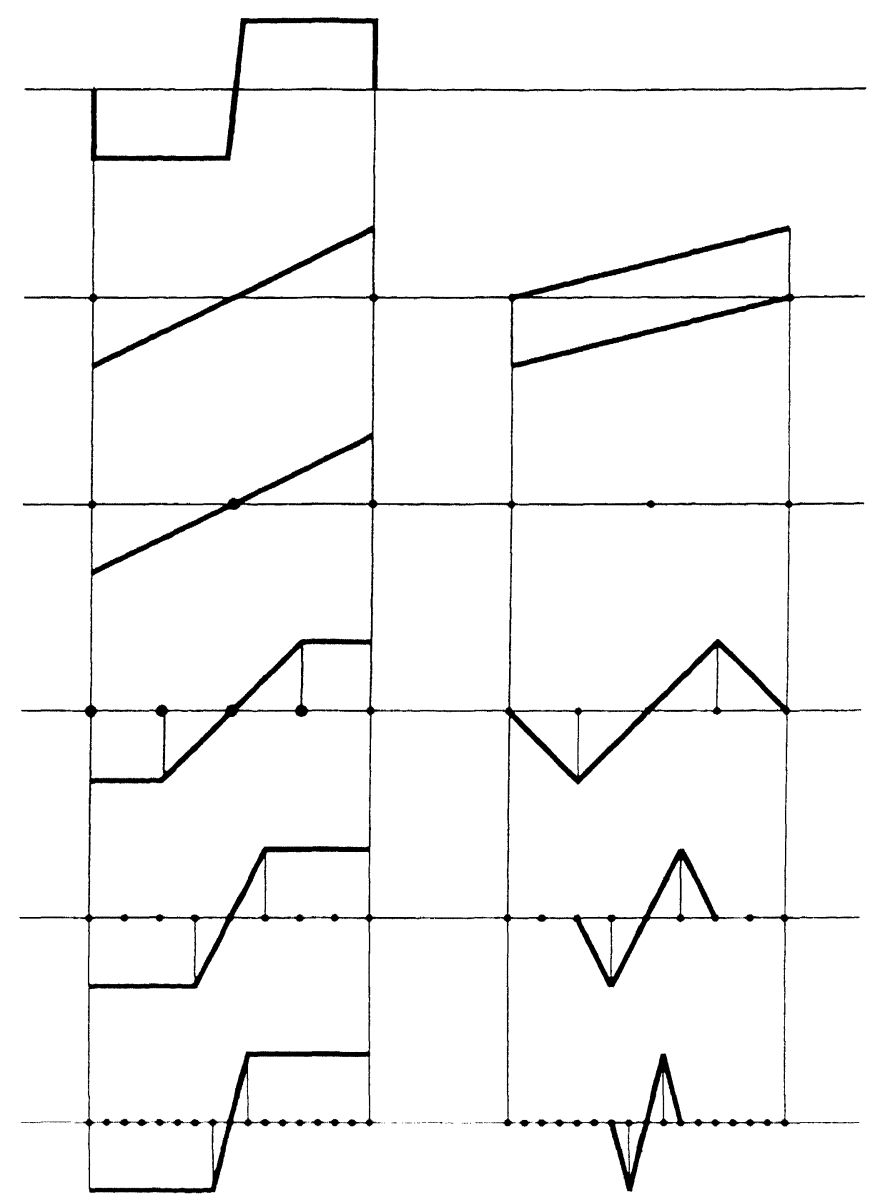

Figure 2. Hierarchical basis decomposition and corresponding standard basis representation for a solution with a large gradient at $x=1 / 2$. The graph at the top shows the approximated function; the graphs at the right show hierarchical contributions to the approximation on the different levels; at the left the successive approximations are shown

Firstly we see that the hierarchical basis component on the grid $\Omega_{1}$ is zero. Without provision, this would result in no further refinement on the grid $\Omega_{2}$. This problem of an early stop for the refinement is due to the counter-symmetric solution. If we use the hierarchical basis components as a criterion for refinement, we should always be aware of this. In the singular perturbation problem in Section 5.1, we do not need particular measures for this, because our solution is strictly monotone.

Secondly, we observe that the size of the hierarchical corrections does not decrease around the jump in the solution. Eventually the refinement will stop when the mesh of the grid $h_{k} \ll \xi^{-1}$, where $\xi$ denotes the slope of the jump in the solution. For true discontinuities refinement will never stop.

In our solution method we do not use the hierarchical basis representation. Nevertheless we use the hierarchical component as a threshold for refinement. In 
the one-dimensional problem we can easily see that the hierarchical surplus on a particular grid $\Omega_{k}$ is easily calculated. Similar, in the two-dimensional case we can use the local hierarchical surplus (12). However, the solutions on the different grids are only consistent up to discretization error. Therefore, used with the discretizations on (subsets) of the regular grids $\Omega_{\mathbf{k}}$, the local hierarchical surplus is not strictly the same as the regular hierarchical decomposition. Nevertheless, on sufficiently refined grids the solutions correspond, except for the (sufficiently small) discretization error.

\subsection{Monotonicity}

Generally, interpolation by means of bilinear basis functions on a grid $\mathscr{G}_{\text {or }} \mathscr{G}_{\ell}$ is not monotonicity preserving. Therefore, the use of the local hierarchical surplus can cause problems when monotonicity is expected. Especially when we consider higher-dimensional problems with relatively large gradients, which are not aligned with the grid, interpolation of monotone functions on the regular grids does not guarantee a monotone result.

Figure 3 shows an example which is likely to appear in the problem studied in Section 5.1. We observe that all values on the three coarsest grids are non-negative, whereas the locally extrapolated value in the dyadic point $\Omega_{\mathrm{kj}}^{+}$on the finest grid is negative. From the example we see that non-monotonic results originate from the use of (10), even when all interpolants are monotone. Further we see that in Fig. 3 the approximations on $\Omega_{\mathbf{k}-\mathbf{e}_{1}}$ and $\Omega_{\mathbf{k}-\mathbf{e}_{2}}$ are consistent, so that the same problem arises if we use hierarchical bases.

A way to circumvent the problem with monotonicity might be by using lower order interpolation. However, we did not further pursue this. Instead we try to alleviate this problem by using sufficiently fine grid refinements.
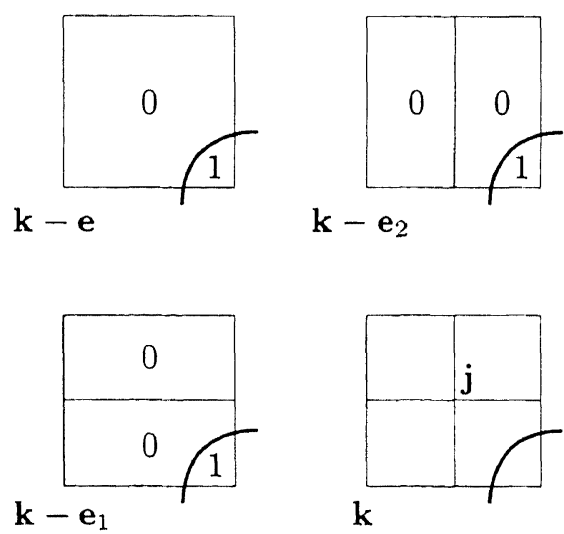

Figure 3. Example of the calculation of the local extrapolated surplus, with a non-monotonic result. Highly irregular but monotone function leads to a non-monotone extrapolated function at point $\Omega_{\mathrm{kj}}^{+}$, shown in the center of the grid $\Omega_{k}$. The interpolant on $\Omega_{k-e}$ is monotone; the corrections from $\Omega_{k-e_{i}}$ and $\Omega_{\mathrm{k}-\mathbf{e}_{2}}$ destroy this monotonicity 
Notice that the monotonicity problems in Fig. 3, which appear if we want to calculate the value in point $\Omega_{\mathrm{kj}}^{+}$, are caused by the two-dimensional local extrapolation. The same problem appears with (12). This local hierarchical surplus is used as a threshold for the refinement criterion. When we calculate the hierarchical surplus based on a one-dimensional extrapolation, e.g., an extrapolation from cell $\Omega_{\mathbf{k}-\mathbf{e}}$ to the cell $\Omega_{\mathbf{k}-\mathbf{e}_{1}}$, with $\|\mathbf{k}-\mathbf{e}\|=0$, see Fig. 3 , the problems with monotonicity do not show up. These one-dimensional extrapolations are always used when we calculate the hierarchical surplus for the grids with $\|\mathbf{k}\|=0$. In our refinement strategy we use this consideration.

\subsection{Refinement Criterion}

We use the local hierarchical surplus as a threshold for the refinement. The singular perturbation problem in Section 5.1 contains steep gradients. As the steep gradients eventually are continuous, this guarantees that the refinement based on a hierarchical surplus calculated with a one-dimensional local extrapolation, eventually stops. Therefore we let the maximum $x$-level and the maximum $y$-level be controlled purely by the refinement based on a one-dimensional calculated hierarchical surplus, i.e., when $\|\mathbf{k}\|=0$. We now use the following criterion for refinement of a particular cell $\Omega_{\mathrm{kj}} \subset \mathscr{G}$.

- If the local hierarchical surplus (12), $\left|\tilde{u}_{\mathrm{kj}}\right|>\delta$, then

If $\|\mathbf{k}\|=0$ then $\mathbf{m}=\max (\mathbf{m}, \mathbf{k}+\mathbf{e})$;

$$
\begin{aligned}
& r_{1}:=\left(\mathbf{m} \geq \mathbf{k}+\mathbf{e}_{1}\right) \\
& r_{2}:=\left(\mathbf{m} \geq \mathbf{k}+\mathbf{e}_{2}\right) .
\end{aligned}
$$

- For $i=1,2$, if $\left(r_{i}\right)$, then create $\mathscr{K}^{\mathbf{e}_{i}}\left(\Omega_{\mathbf{k j}}\right)$.

In the current situation, $\mathbf{m}$, the multi-integer defining the maximum $x$-level and the maximum $y$-level, is initially set to $\mathbf{m}=\mathbf{0}$. Further, the magnitude of $\mathbf{m}$ is controlled by the finest cells $\Omega_{\mathbf{k j}} \in \mathscr{G}$, with $\|\mathbf{k}\|=0$. So the maximum $x$-level and the maximum $y$-level, denoted by the vector $\mathbf{m}$ are set when $\|\mathbf{k}\|=0$, and hence $\mathbf{m}$ is purely controlled by refinement based on one- dimensional extrapolation.

\section{Numerical Results}

\subsection{The Test Problem}

As a test problem we consider a singular perturbation problem in two dimensions proposed in [9]. The original problem was defined on an unbounded domain in $\mathbb{R}^{2}$. For its numerical solution we truncate the domain of definition to a sufficiently large rectangle, and because of the problem's symmetry, we only approximate the solution in half of the proposed domain. Thus, the problem we solve is given by

$$
u_{x}-\varepsilon \Delta u=0
$$




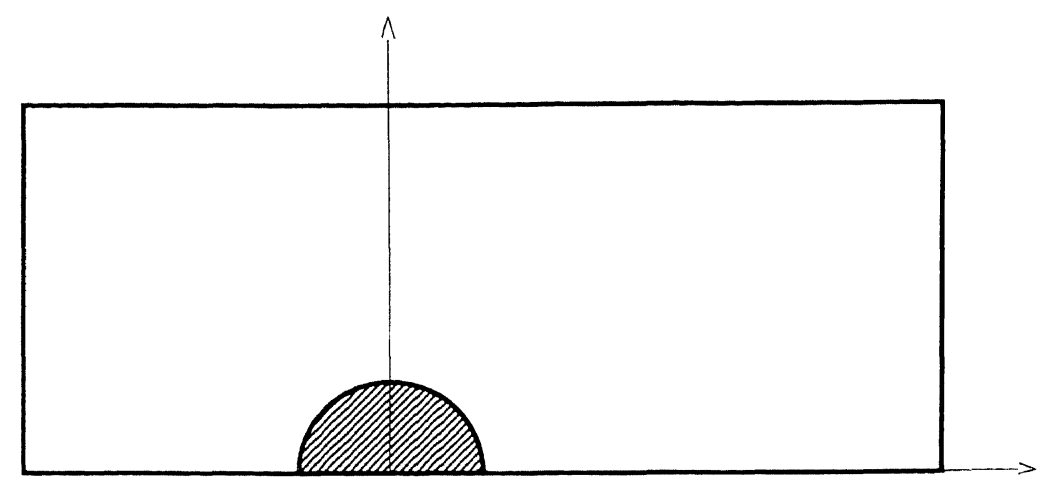

Figure 4. Domain of the singular perturbation problem

on a rectangular domain $\Omega=\left(N_{L}, N_{R}\right) \times\left(0, N_{T}\right) \backslash\left\{(x, y) \mid x^{2}+y^{2} \geq 1\right\}$, with $N_{L}, N_{R}$, and $N_{T} \in \mathbb{N}$, and $0<\varepsilon \ll 1$, (see Fig. 4). We solve the numerical problem with $\Omega=(-4,6) \times(0,4)$, so that on the coarsest mesh $S=(10,4)$. An analytical solution for the original problem on the unbounded domain is given in [9].

On the bounded domain we apply the following boundary conditions:

$$
\begin{gathered}
x^{2}+y^{2} \leq 1 \rightarrow u(x, y)=1, \\
y \in\left[0, N_{T}\right] \rightarrow u\left(N_{L}, y\right)=0, \\
y \in\left[0, N_{T}\right] \rightarrow u_{x}\left(N_{R}, y\right)=0, \\
x \in\left[N_{L}, N_{R}\right] \rightarrow u_{y}\left(x, N_{T}\right)=0, \\
x \in\left[N_{L}, N_{R}\right] \rightarrow u_{y}(x, 0)=0 .
\end{gathered}
$$

On each grid $\Omega_{\mathbf{k}}$ Eq. (16) is discretized by first-order upwind differencing. At the dyadic point $\Omega_{\mathbf{k j}}^{+}$the discrete equation is written as,

$$
\frac{u_{l}-u_{c}}{h_{l}}+\varepsilon \frac{\frac{u_{r}-u_{c}}{h_{r}}-\frac{u_{c}-u_{l}}{h_{l}}}{\left(h_{r}+h_{l}\right) / 2}+\varepsilon \frac{\frac{u_{t}-u_{c}}{h_{l}}-\frac{u_{c}-u_{b}}{h_{b}}}{\left(h_{t}+h_{b}\right) / 2}=0,
$$

where the parameters $h_{l}, h_{r}, h_{t}, h_{b}, u_{c}, u_{l}, u_{r}, u_{t}$, and $u_{b}$ take different values in the different situations, depending on the grid $\Omega_{\mathrm{k}}$ and the position of $u_{c}$ in the domain, see Fig. 5. Notice that we consider only rectangular grids. If no boundary points are involved, we have $h_{l}=h_{r}=2^{k_{1}}, h_{t}=h_{b}=2^{-k_{2}}, u_{c}=u_{\mathrm{kj}}$, $u_{l}=u_{\mathbf{k j}-\mathbf{e}_{1}}, u_{r}=u_{\mathbf{k j}+\mathbf{e}_{1}}, u_{b}=u_{\mathbf{k j}-\mathbf{e}_{2}}, \quad u_{t}=u_{\mathbf{k j}+\mathbf{e}_{2}}$. We notice that this first-order accurate discretization will yield a monotone solution on each separate grid $\Omega_{\mathbf{k}}$.

For $\varepsilon \ll 1$ we have a strongly convection-dominated problem and diffusion only plays a role in particular regions. For small $\varepsilon$, the solution will have large gradients in front of the cylinder and there will be a distinct wake at the back. Large gradients, especially when these gradients do not align with the coordinate directions, cause difficulties for standard sparse grids because of the large cross derivatives [10]. To solve the problem which appears with the non-aligned gradient we consider adaptive grids. 


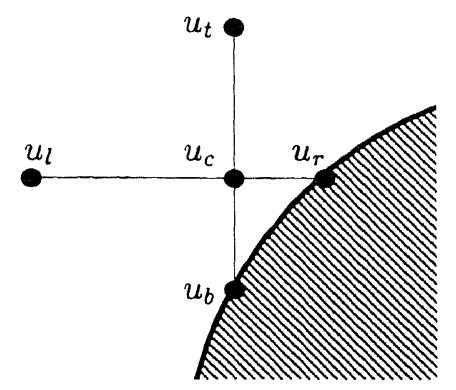

Figure 5. Example of stencil near the circle $x^{2}+y^{2}=1$

\subsection{Regular Combination Solution}

First we compare the solution obtained by the (standard) combination technique applied to $\mathscr{G}_{6}$ with a "reference" solution, calculated on the single grid $\Omega_{(6.6)}$. For these computations we take the small parameter $\epsilon=1 / 50$. No numerical problems are expected on $\Omega_{(6,6)}$ because $\varepsilon=1 / 50>1 / 64=2^{-6}$, which implies that the sharp layers can be completely resolved on this mesh.

Figure 6 shows the reference solution. Figure 7 shows the combination solution, on the sparse family of grids $\mathscr{G}_{\ell}$, with $\ell=6$. We see that the regular sparse grid solution shows monotonicity problems, as mentioned in Section 4.2. The considerations apply because the regular combination formula is derived from subsequent two-dimensional extrapolations.

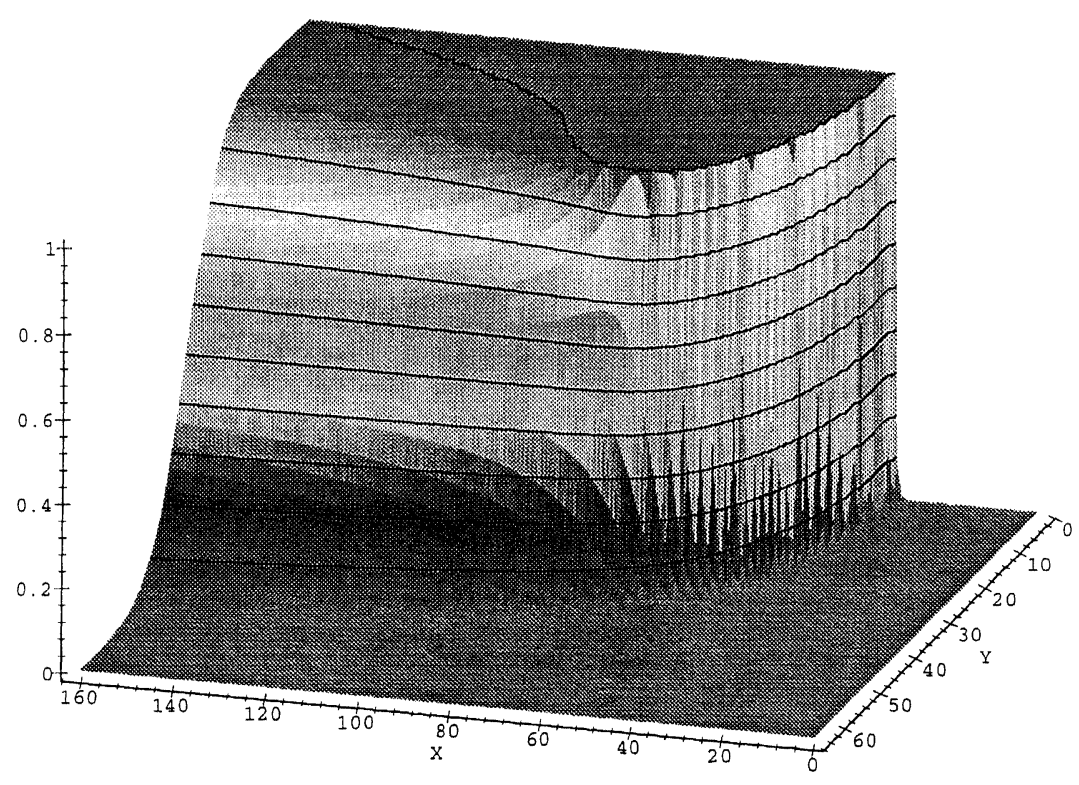

Figure 6. Reference solution on grid $\Omega_{(6,6)}$ 

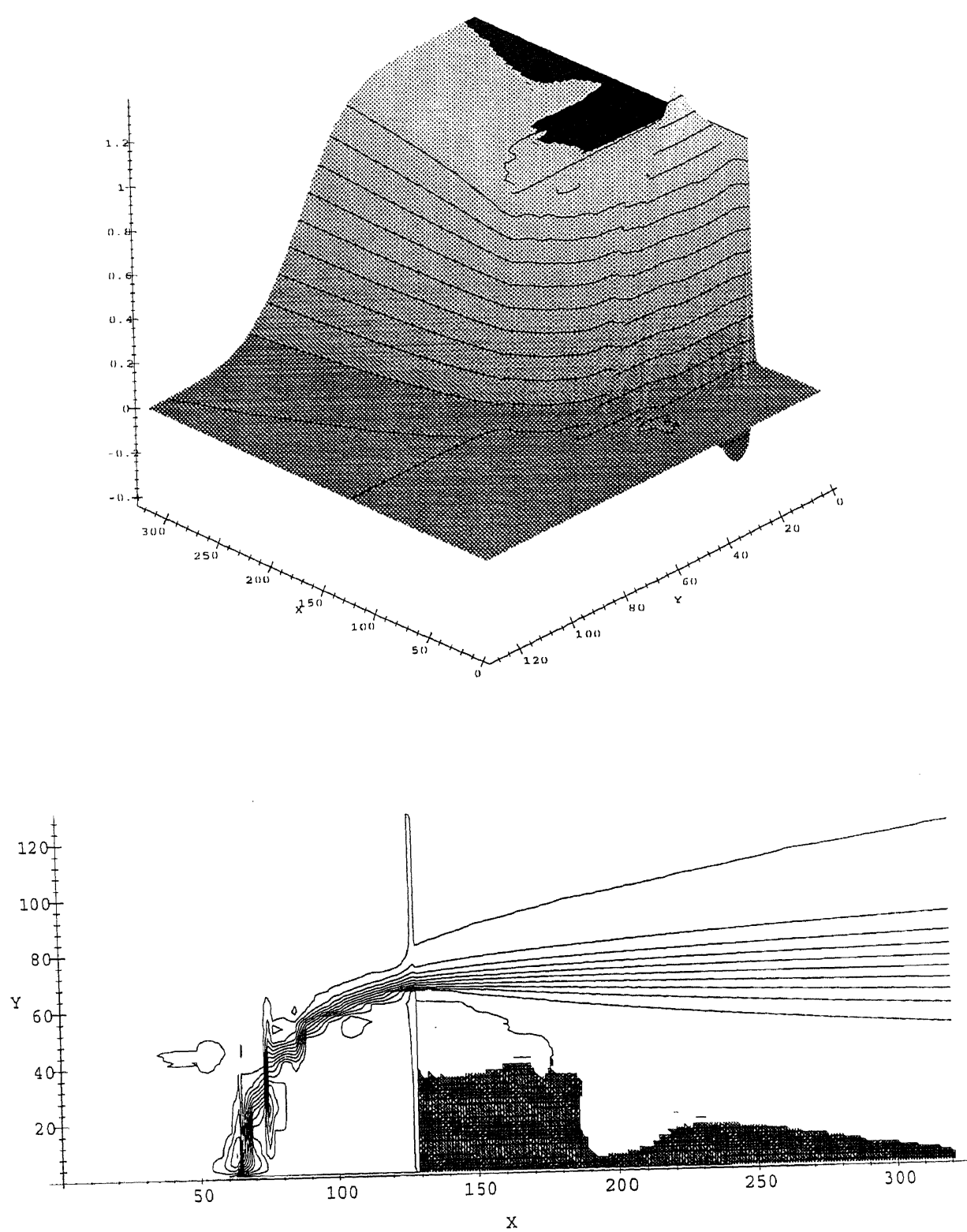

Figure 7. Combination solution from all grids $\Omega_{\mathrm{k}} \subset \mathscr{G}_{\ell}$, with $\ell=6$

Table 1 shows different norms for the difference between the combination solution and the reference solution for various values of $\ell$. For sufficiently smooth solutions we may expect an $\mathcal{O}\left(h^{2} \log (h)\right)$ extrapolation error reduction for the $L_{2}$-norm for the two dimensional problem [10, Thm. 3.6], possibly on top of an $(1)(h)$ discretization error. (However, if a proper multivariate h-expansion exists, then discretization errors may cancel to some extent.) In fact, we see that there is 


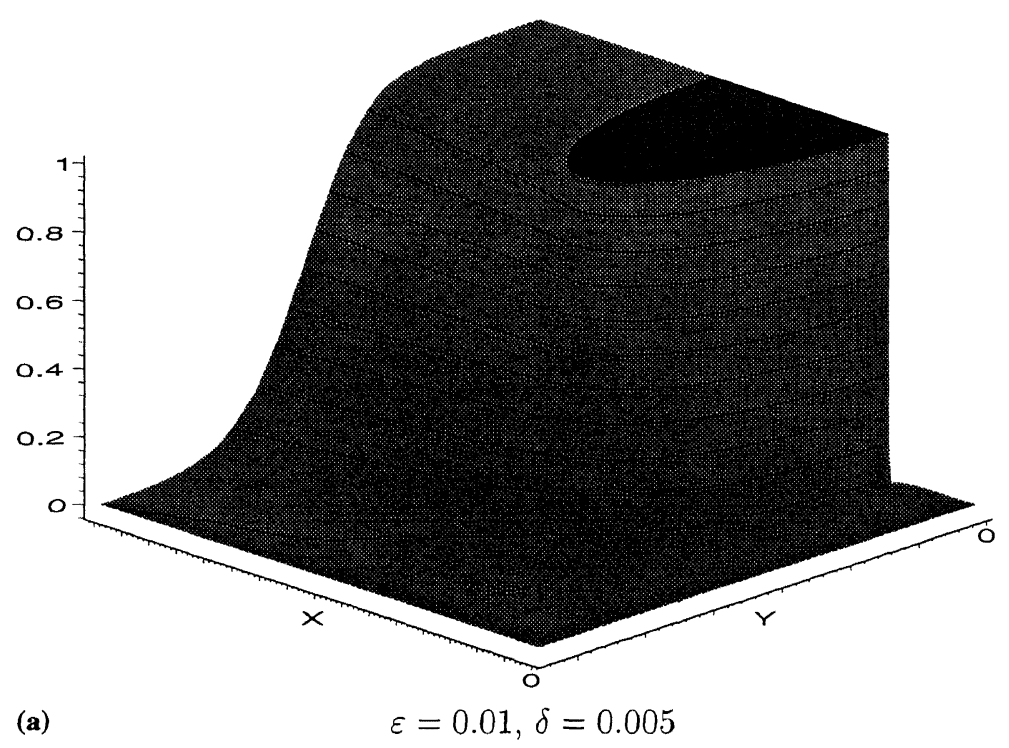

(b)

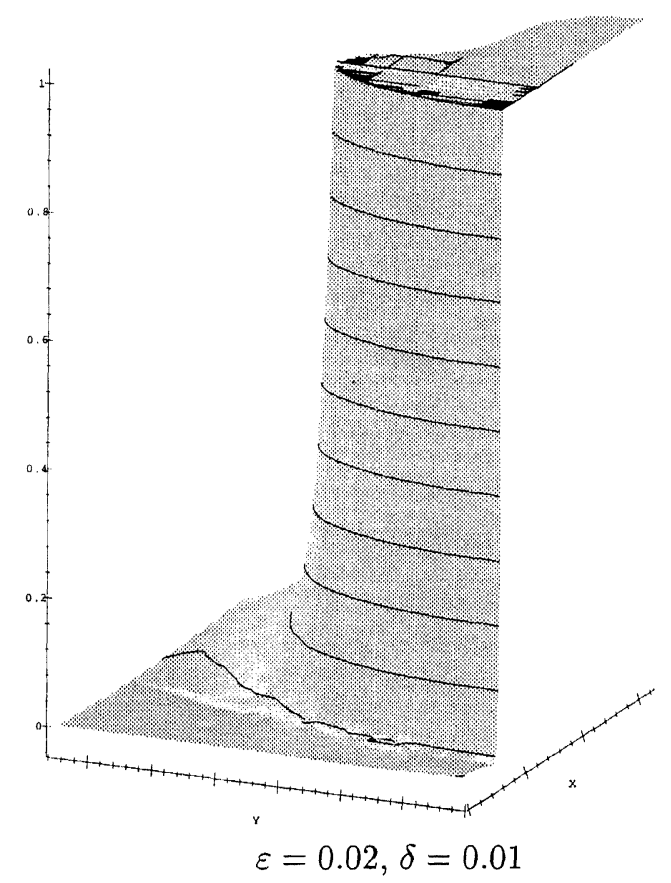

Figure 8. Two views of the adaptive combination solution with small different $\varepsilon$ and $\delta$. The bottom view clearly shows the non-monotonicity in the combination solution. $\mathbf{a} \varepsilon=0.01, \delta=0.005$. $\mathbf{b} \varepsilon=0.02$, $\delta=0.01$ 
hardly any convergence. This again is a result of lack of smoothness in the solution.

Where convergence is slow for the $L_{1}$-norm, no convergence is seen in the $L_{\infty}$ norm. Apparently, the non-monotonicity does not disappear, even for large values of $\ell$. The first effect of the further refinements is that we restrict the overshoots and undershoots to a smaller area. First the maxima of these over- and undershoots are not really suppressed. However, eventually for $\ell$ large enough, convergence will be seen.

\subsection{Adaptive Combination Solutions}

For the computations in this section the same problem is used as in the previous section. However, we now take the small parameter $\varepsilon=0.01$. As a threshold for the refinement criterion as described in Section 4.3 we use $\delta=1 / 200$. By this criterion, on the finer grids $\Omega_{\mathrm{k}}$ the discretized equation is solved (only) on (small) subdomains of $\Omega$. Here the same discretization is used as in Section 5.1 and

Table 1. Nodal error and number of nodes for the combination solution

\begin{tabular}{clllr}
\hline$\Omega_{h}$ & $\|$ error $\|_{\infty}$ & $\|$ error $\|_{2}$ & $\|$ error $\|_{1}$ & \# nodes \\
\hline $\mathscr{G}_{1}$ & $0.8887 \cdot 10^{0}$ & $0.1955 \cdot 10^{0}$ & $0.1076 \cdot 10^{0}$ & 457 \\
$\mathscr{G}_{2}$ & $0.8379 \cdot 10^{0}$ & $0.1265 \cdot 10^{0}$ & $0.6185 \cdot 10^{-1}$ & 1038 \\
$\mathscr{G}_{3}$ & $0.8460 \cdot 10^{0}$ & $0.8982 \cdot 10^{-1}$ & $0.3614 \cdot 10^{-1}$ & 2532 \\
$\mathscr{G}_{4}$ & $0.8921 \cdot 10^{0}$ & $0.7448 \cdot 10^{-1}$ & $0.2210 \cdot 10^{-1}$ & 6171 \\
$\mathscr{G}_{5}$ & $0.8851 \cdot 10^{0}$ & $0.5648 \cdot 10^{-1}$ & $0.1297 \cdot 10^{-1}$ & 14739 \\
$\mathscr{G}_{6}$ & $0.8032 \cdot 10^{0}$ & $0.3810 \cdot 10^{-1}$ & $0.7844 \cdot 10^{-2}$ & 34444 \\
$\Omega_{(6,6)}$ & & & & 164737 \\
$\mathscr{G}_{\mathscr{G}_{6}} \mathscr{G}_{6}$ & $0.7967 \cdot 10^{0}$ & $0.3974 \cdot 10^{-1}$ & $0.1380 \cdot 10^{-1}$ & 6077 \\
\hline
\end{tabular}

The discrete solution on the regular grid $\Omega_{(6,6)}$ serves as the reference solution for the singular perturbation problem (16) with $\varepsilon=1 / 50$. Bottom line: nodal error of the adaptive combination solution

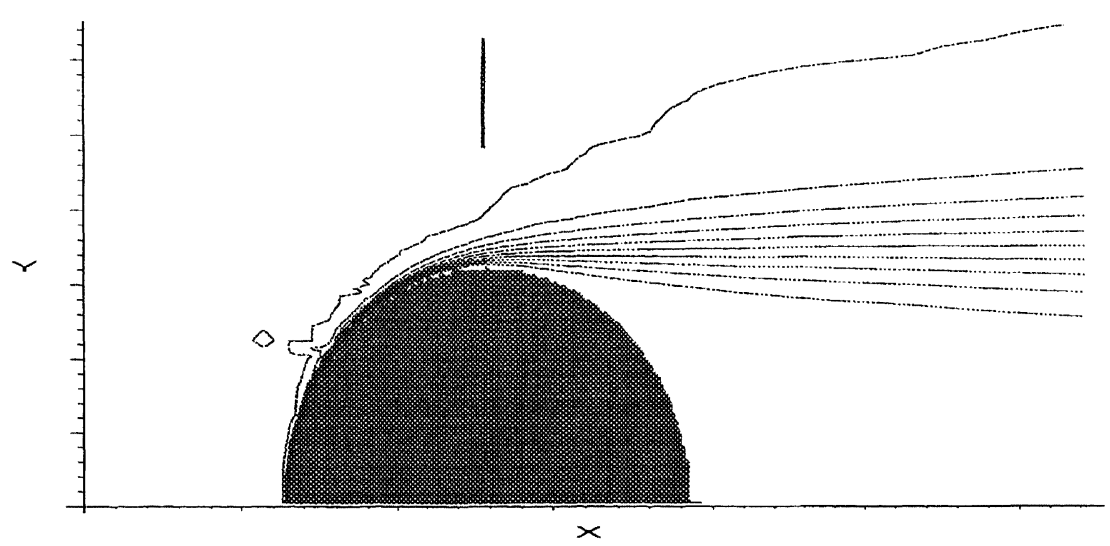

Figure 9. Contours of combination solution with $\varepsilon=1 / 100$ and $\delta=1 / 200$ 


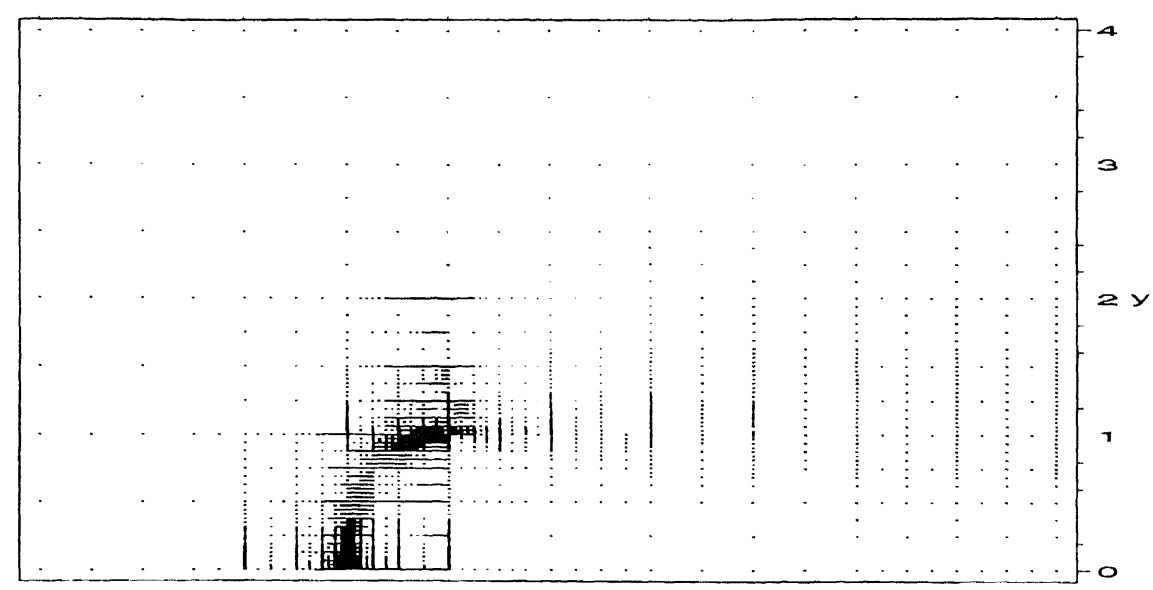

Figure 10. Adaptive sparse grid for the solution with $\varepsilon=1 / 100$ and $\delta=1 / 200$

(artificial) boundaries of these subdomains use Dirichlet boundary conditions. The necessary boundary values are derived from the coarser grid solutions by means of the adaptive combination formula (15).

The number of points for the solution satisfying this criterion is approximately 22250 and the "finest" grid $\Omega_{\mathbf{k}} \in \mathscr{G}$ is $\mathbf{k}=(12,7)$, so the highest level is $\ell=19$. The minimal and maximal values of the solution as shown in Fig. 8 are $\left(u_{\min }, u_{\max }\right)=(-0.0239,1.0)$. The fact that no overshoot is seen is partly due to the treatment of the boundary conditions, i.e., the code to calculate the solution for $\mid \mathbf{x} \leq 1.0$. The undershoot is of the order of $\delta$, which is acceptable. The position of the minimal solution is $\mathbf{x}=(-0.3125,1.50)$. Figure 9 shows the contour lines of this solution. In Fig. 10 the adaptive sparse grid $\Omega_{\mathbf{k j}}^{+} \in \mathscr{G}$ is shown.

\section{Conclusions}

In this paper we introduce an adaptive combination formula for the computation of the solution of a PDE on an adaptive sparse grid. We apply the formula to solve a two-dimensional singular perturbation problem of which the solution has both boundary and interior layers. The $\left(1 \varepsilon^{1 / 2}\right)$ interior layers, that are aligned with the grid, are efficiently approximated by the adaptive semi-refinement. The ( $(\varepsilon)$ boundary layer, that is not particularly aligned, is nevertheless well captured by a fine, locally almost regular grid.

\section{In memoriam}

Jaap Noordmans, the first author of this paper, died July 23, 1999, after several years of illness. He was much devoted to the subject of sparse grids and he worked with much enthusiasm on this paper, that was meant to become a part of his PhD thesis. He prepared all computations and figures shown. We discussed the draft paper until a month before his 
death. We all admire the way he fought his illness, and we very much miss his person and his enthusiastic cooperation.

\title{
References
}

[1] Bungartz, H.-J.: An adaptive Poisson solver using hierarchical bases and sparse grids. In: Iterative methods in linear algebra (Beauwens, R., ed.), pp. 293-310. Amsterdam: North-Holland 1992.

[2] Bungartz, H.-J., Griebel, M., Rüde, U.: Extrapolation, combination, and sparse grid techniques for elliptic boundary value problems. Comput. Methods Appl. Mech. Eng. 116, 243-252 (1994).

[3] Everaars, C. T. H., Koren, B.: Using coordination to parallelize sparse-grid methods for 3D CFD problems. Parallel Comput. 24, 1081-1106 (1998).

[4] Griebel, M.: A parallelizable and vectorizable multi-level algorithm on sparse grids. In: Parallel algorithms for partial differential equations. Notes Numer. Fluid Mech. 31, pp. 94-100. Wiesbaden: Vieweg, 1991.

[5] Griebel. M.: The combination technique for the sparse grid solution of PDEs on multiprocessor machines. Parallel Proc. Lett. 2, 61-70 (1992).

[6] Griebel, M.: Adaptive sparse grid multilevel methods for elliptic PDEs based on finite differences. Computing 61, 151-180 (2000).

[7] Griebel, M., Huber, W., Rüde, U., Störtkuhl, T.: The combination technique for parallel sparsegrid-preconditioning and -solution of PDEs on multiprocessor machines and workstation networks. In: Parallel processing (Bouge, L., Cosnard, M., Robert, Y., Trystram, D., eds.), pp. 217-228. Lecture Notes in Computer Science 634. Berlin Heidelberg New York Tokyo: Springer, 1992.

[8] Griebel, M., Huber, W., Störkuhl, T., Zenger, C.: On the parallel solution of 3D PDEs on a network of workstations and on vector computers. Lecture Notes in Computer Science. 732, 276291 (1993).

[9] Hemker, P. W.: A singularly perturbed model problem for numerical computation. J. Comp. Appl. Math. 76, 277-285 (1997).

[10] Hemker, P. W., Pflaum, C.: Approximation on partially ordered sets of regular grids. Appl. Numer. Math. Trans. IMACS 25, 55-87 (1997).

[11] Pflaum, C., Rüde, U.: Gauß adaptive relaxation for the multilevel solution of partial differential equations on sparse grids. J. Comput. Inf. 4, 55-71 (1993).

[12] Störtkuhl, T.: Ein numerisches adaptives Verfahren zur Lösung der biharmonischen Gleichung auf dünnen Gittern. PhD thesis, Technische Universität München, 1995.

[13] Zenger, C.: Sparse grids. In: Parallel algorithms for partial differential equations (Hackbusch, W., ed.), pp. 297-301. Notes on Numerical Fluid Mechanics 31. Braunschweig: Vieweg, 1991.

\author{
P. W. Hemker \\ Centrum voor Wiskunde en Informatica \\ P.O. Box 94079 \\ NL-1090 GB \\ Amsterdam \\ The Netherlands \\ e-mail: P.W.Hemker(ucwi.nl
}

Verleger: Springer-Verlag KG, Sachsenplatz 4-6, A-1201 Wien. - Herausgeber: Prof. Dr. Wolfgang Hackbusch, Institut für Informatik und Praktische Mathematik, Christian-Albrechts-Universität, Olshausenstraße 40, D-24098 Kiel. - Satz. und Umbruch: Scientific Publishing Services (P) Ltd., Madras; Offsetdruck: MANZ Crossmedia, A-1051 Wien. Verlagsort: Wien. - Herstellungsort: Wien. - Printed in Austria. 